\title{
Inhalt der ersten Hälfte der Fortschritte der Physik 1871.
}

\author{
Erster Abschnitt: Allgemeine Physik.
}

1. Maass nnd Messen: Steinheil 3. - Karsten 4. - Das Metermaass in Oesterreich 5. - Tinter 6. - Gesetz zur Einführung des Metermaasses in England 8. - L. Levi 8. - Comitébericht über die Einführung des metrischen Maasssystems 9. - Comitébericht der British Association über metrisches Maasssystem 9. - F. Hermann 10. - Bornemann 10. - Kubiktabellen für Metermaass 11. - Oberbeck 12. - Köpke 13. - Ch. H. Dowling 14. - v. d. Willigen 14. - Stamkart und Stuart 14. - C. Maxwell 15. - W. Mann 16. - C. Rodenbach 16. - ${ }^{*}$ G. Govi 17. - F. J. Stamkart 17. - Europäische Gradmessung 19. - De Magnac 20. - G. W. Dean 21. - W. Thomson 22. - G. B. Airy 22. - Clarke 23. - H. Fritsche 23. - Watkin 24. - Paschwitzscher Distanzmesser 25. - Heuser 26. - J. Plucker 27. - C. Naumann 28. - E. A. Matzenauer 28. - Millar's Höhenmessapparat 28. - Bauernfeind 29. - Bauernfeind 30. - H. H. Heys van Zouteveen 31. - Combes 31. E. Deprez 32. - Exter's Geschwindigkeitmesser 32. - Herder 33. - G. W. Hough 34. - Edelmann 35. - Secchi 36. - F. J. Stamkart 36. - Zenger 38. - Pickering 39. - L. Lipkin 40. - E. Weiss 41. - Hilgard 42. Phillips 42. - Phillips 43, - E. Wensch 44. - v. Schlagintweit-Sakünlünski 44. - Reinsch 46. - Fernere Litteratur 46.

2. Dichtigkeit: F. J. Stamkart 47. - A. R. v. Schrötter 48 . - H. L. Buff 49. - B. Tollens 49. - E. Zettnow 49. - Scott 49. - J. Y. Buchanan 49. - H. Ludwig 50. - A. Bauer 50. - C. A. Valson 51. - Marignac 51. - A. Schröder 53. - Rieth 53. - R. A. Wright 53. - B. Franz 54. White Hodding 55. - M. Mach 55. - L. Carius 55. - Fernere Litteratur 56.

3. Moleknlarphysik: Crookes 59. - W. Thomson 59. - A. Horstmann 59. - Peslin 60. - A. Naumann 60. - A. Naumann 61. - L. Pfaundler 61. - Beketoff und Czernay 62. - H. C. Dibbits 62. - F. W. Krecke 63. J. W. Gunning 63. - F. W. Krecke 63. - Berthelot 64. - Pfaff 64. F. Mohr 65. - Gladstone und Tribe 65. 66. - W. Müller (Perleberg) 68. Baudrimont 70. - Lemoine 70. - Groshans 72. - W. Zängerle 73. - Ditte 73. - Kaufmann 73. - A. H. Church 74. - A. Schertel 74. - v. Schrötter 74. - T. G. Bonney 75. -- A. Forster 75. - Klocke 76. - A. Marschall 76. - R. Mallet 76. - L. Meyer 77. - G. Rose 77. - G. Wunder 77. A. Knop 77. - H. Vogelsang 78. - E. Weiss 78. - Schlösing 78. - C. Marangoni u. P. Stefanelli 78. - Fernere Litteratur 79.

4. Mechanik: W. M. W. 85. - J. D. Everett 85. - R. Clansius 85. E. Zetzsche 87. - R. S. Ball 87. - J. Bertrand 88. - M. Chasles 88. G. Bardelli 88. - H. Airy 88. - W. Swan 88. - Drach 88. - *A. S. Davis 88. - H. Highton 88. - Ungleichheiten der Mondbewegung 88. - Bashforth 89. - W. H. A. Russel 89. - G. Krebs 89. - N. M. Ferrers 90. - J. A. Serret 90. - J. Curie 91. - E. Rolland 91. - H. Résal 91. - Phillips 91. - S. New comb 91. - P. A. Hansen 92. - K. Culmann 93. - G. Hagen 93. - H. Résal 94. - H. Gyldén (2 Abhandl.) 94. - Bazin 95. - A. Kurz 95. - M. Rankine 95. - J. A. Grunert (2 Abhandl.) 96. - J. C. Maxwell 96. - J. Petersen 97. - G. R. Dahlander 97. - W. Matzka 97. - D. Piani 97. - Stroumbo 98. - Dauplay 98. - J. D. Everett 98. - Muir 98. H. Haedicke 98. - B. Stauffer 98. - J. Klein 99. - Fernere Litteratur für 1871. 99. - Mathematischer Litteraturnachtrag für 1870. 101. - Derselbe lür 1871. 103. - M. Rankine (1870) 104. - C. Oechsle (1870) 105. - L. Saalschïtz (1870) 105. - F. Jenkin 105. - Breton (1870) 106. - Fernere Litteratur 106 . referirt.

Anmerkung. Die mit einem * versehenen Abhandlungen sind nicht

Fortschr. d. Phys. XXVII. 
5. Hydromechanik: Rühlmann 108. - De St.-Venant 112. - Bertin 115. - Fargne 118. - Vogelsang 120. - Airy's Untersuchungen 121. - Knowles 121. - G. Schmidt 122. - Tommasi's Fluthmaschine 123. - Condurier 123. - Nenf u. Dumont 124. - Coates u. Lassell 124. - Chameroy's Leitungshahn 124. - Chameroy's Apparate etc. 125. - Virtue 125. - J. A. Mueller (Amsterdam) 126. - Brocard's Wassermesser 126. - Piezometrische Wassermessapparate 127. - Zettnow 127. - Leeds 127. - De Koninck 127. - Caligny 128. - Zaliwsky 128. - Emmanuel 129. - Röhrs 130. - A. Moll 130. - I. Boltzmann 130. - J. Todhunter 131. - Rankine 132. - H. Moseley (2 Abhandl.) 133. - W. Thomson 134. - Partiot 135. - De St.-Venant 135. - J. Boussinesq (2 Abhandl.) 138. - J. Boussinesq (2 Abhandl.) 140. - Colding 141. - Barthélemy 142. - Zuschlag 143. - G. Hagen 145. - Fernere Litteratur 149.

6. Aểrodynamiin: A. Heller 152. - A. Kurz 153. - H. Wild 154. M. Hall 155. - Amagat 155. - V. Regnault 156. - K. H. Schellbach 158. - R. St. Ball 160. - *Niemeitz 160. - v. Lang (2 Abhandl.) 161. J. Stefan 163. - O. E. Meyer 168. - Labrousse 174. - De Fonvielle 174. - Janssen 174. - Bourdin 174. - Serret 174. - Janssen 175. - Janssen 176. - De Fonvielle 177. - Tastes 177. - H. Dorner 177. - Fernere Litteratur 178.

7. Cohåsion und Adhăsion: A. Festigkeit und Elasticität: De St.Venant 180. - C. Douglas 181. - R. Hoppe 181. - J. Wolf 182 . H. Tresca 183. - F. Schott 183. - W. H. Johnson 184. - Brockbank, Joule, Spence 184. - Joule 184. - Brockbank 184. - Fairbairn, Joule, Spence 184. - Federmanometer 185. - Michele u. Carrington 185. - Alcock 185. - De St. - Venant 186. - Levy 186. - Schmulewitsch 186. - Heppel 188. M. Rankine 188. - Barlow 188. - ${ }^{*}$ W. Thomson 188. - Schneebeli (3 Abhandl.) 188. - ${ }^{*}$ Schneebeli 191. - ${ }^{*}$ E. Villari 191. - Fairbairn 191. Tilghman 191. - J. Boussinesq 192. - De St.-Venant 193. - Widerstandsfähigkeit von Röhren 194. - G. Bischof 194. 195. - J. Syme 195. - Dumas 195. - Montefiore-Levi u. C. Künzel 196. - 'Tresca 196. - De St.-Venant 197. - Tresca 197. - R. Hoppe 198. - Ueber eine Abhandlung Levy`s 199. - Fernere Litteratur 200.

B. Capillarität: E. Roger 202. - C. A. Valson 203. - J. Moutier 204. - O. Loew 205. - J. Kober 206. - J. Platean 207. - Fernere Litteratur 207.

C. Löslíchkeit: C. Tuchschmidt u. C. Follenius 208. - A. Seely 208. - Aguiar u. A. Bayer 209. - Wartha 209. - Jacobsen 209. - G. Leuchs 209. - F. Schulze 210. - F. Mohr, L. Buchner 210. - De Coppet 210. F. Pfaff 211. - F. Wöhler 211. - F. Sestini 212. - A. Anderssohn 212. Stas 212. - Is. Pierre 212. - R. Warrington 213. - Sestini 213. - F. A. Flückiger 213. - Valson 214. - Henrici 215. - Tomlinson 216. - S. SoJaro 216. - Merget 217. - J. Benigar 218. - Stefan 220. - F. Mohr 220. - Fernere Litteratur 221.

D. Absorption: J. Hunter 223. - G. Bischof jun. 223. - Mallet 223. - J. Phillips 223. - R. Böttger (2 Abhandl.) 224. - H. Kolbe 224. - Bretschneider 225. - Scheermesser 225. - Tyndall's Respirator 227. - Fernere Litteratur 227.

E. Adhäsion. -

\section{Zweiter Abschnitt: Akustik.}

8. Physikalische Akustik: K. H. Schelibach 231. - K. H. Schellbach 234 . - Ghallis 235. - F. Guthrie 238. - A. Seebeck 243. - J. J. Oppel 243. H. Schneebeli (2 Abhandl.) 248. - H. Schneebeli 248. - H. Planeth 249. J. J. Müller 250. - A. 'Terquem 253. - J. F. Boutet 256. - A. Cornu u. E. Mercadier 271. - Bourget 274. - J. Bourget 277. - E. Gripon 279. E. Gripon 280. - W. Fonvielle 281. - W. Fonvielle 282. - A. Obermayer 283. - Govi 284. - Barry 284. - D. Joseph 285. - C. J. Schallgeschwin- 
digkeit in Kohle 285. - Ballo 285. - J. Moutier 286. - Fernere Litteratur 286.

9. *Physiologisclie Aknstlk 288 . Vergleiche Jahrgang 1870 p. 962.

\section{Dritter Abschnitt: Optik.}

10. Theorie des Lichts: Ed. Ketteler 291. - Cornu 295. - J. W. Strutt 298. - J. W. Strutt 302. - J. W. Strutt 307. - A. Töpler 309. - Fernere Litteratur 315 .

11. Fortpflanzung, Spiegelung und Brechung des Lichts: C. Christiansen 316. - A. Kundt (4 Abhandl.) 318. - J. L. Soret 321. - v. Lang 322. F. Kohlrausch 322. - W. Sellmeier 322. - J. Stefan 323. - Blaserna 324. - J. I. Sirks 324. - W. Wernicke 325. - C. Schultz - Sellack 327. - Andrews 328. - O. N. Rood 328. - Hansen 328. - F. Mohr 329. - F. Mohr 330. - v. d. Willigen 330. - v. Kerkhoff 331. - Fernere Litteratur 331.

12. Objektive Farben, Spektrum, Absorption: J. Browning 333. - Blaserna 333. - Stoney 333. - Stoney u. Reynolds 333. - Soret 335. - Stoney 336.

- F. Zöllner (2 Abhandl.) 336. - Ángström 340. - Wüllner 341. - Weinhold 345. - Soret 345. - Draper's Experiment 346. - Zenker 346. - W. de la Rue 346. - Huggins 346. - Secchi 347. - Secchi 348. - Proctor 348. - Secchi 349. - Secchi 350. - Sechi 350. - Janssen 352. - Janssen 353. - Rayet 353. - C. A. Young 355. - C. A. Young 355. - F. Zöllner 356. - F. Zöllner 358. - F. Zöllner 362. - Seabroke 363. - H. Vogel 363. Huggins 365. - Huggins 366. - H. Vogel 366. - Talbot 366. - Zöllner 367. - J. Browning 367. - Ellery 367. - Lindsay 368. - Procter 368. T. W. Backhouse 368. - C. P. Smyth 368. - Börgen u. Copeland 369. A. Herschel 369. - C. P. S. 369. - Barker 370. -- Janssen 370. - Hennessey 371. - H. Vogel 371. - Ellery 372. - Förster 372. - Börgen u. Copeland 373. - Troost u. Hautefeuille 373. - G. Salet 374. - Ed. Becquerel 374. - Salet 374. - Salet 376. - Ditte 376. - Dumas 376. - Ditte 376. - Lecoq de Boisbaudran 378. - Lecoq de Boisbaudran 379. - Papillon 379. - Cornu 380. - Janssen 380. - Houston 381. - Meusel 381. - Böttger 381. - A. Vogel 382. - W. Stein 382. - Blochmann 383. - v. Kobell 384. - Blaserna 385. - Watts 385. - Snelns 386. - Kerpely 386. - Williams 387. - Roscoe 387. - Spear Parker 388. - Seely 389. - Erk (2 Abhandl.) 389. - Carl 389. - Salleron's Colorimeter 390. - Rheineck 390. - Vierordt 391. - Preyer 391. - Vierordt 391. - H. Schiff 391. - E. Lommel 392. E. Lommel 393. - Campani 393. - Andrews 394. - Sorby 394. - Schönn 395. - J. J. Müller 395. - E. Lommel 396. - Fernere Litteratur 398.

13 Photometrie: Vierordt 403. - Carl 404. - Roscoe u. Thorpe 404. Zenger 405 . - Fernere Litteratur 406.

14. Phosphorescenz und Fluoresçenz: Alvergniat 408. - Forster 408. Jousset 409. - Panceri 409. - E. Lommel 410. - Obermann 412. - Baeyer 413. - Tsehermak 413. - Le Comte du Moncel 413. - Morton 413. Fernere litteratur 414.

15. Interferenz, Polarisation, Doppelbreclung, Krystalloptlk: Ditscheiner 414 . - E. Jochmann 419. - G. Quincke 421. - Crova 435. - Ditscheiner 436. - Ditscheiner 43̊8. - J. L. Sirks 439. - Mascart 441. - H. W. Dove 442. - J. Müller 442. - A. Kurz 443. - Deas 443. - Maxwell 443. - Wheatstone 443. - Schultz-Sellack 444. - Adams 444. - Strutt 445. - Litteratur 445. - P. Groth 446. - G. Tschermak 448. - Brezina 449. - Fernere Litteratur 450 .

A. Circularpolarisation: Croullebois 450. - Jegorow 452. - Gill 453. - O. Popp 453. - Fernere Litteratur 453.

16. Chemische Wirkungen des Lichts : C. Schultz-Sellack 454. - C. SchultzSellack 455. - C. Schultz-Sellack (2 Abhandl.) 457. - H. Vogel 458. E. Budde 459. - H. Vogel 460. - A. Vogel 460: - F. Gintl 460. - Swan 461. - Raoult 461. - Swan 461. - Simpson 461. - Monkhoven 462. - 
Vidal 462. - Selmi u. Piacenti 463. - Schnauss 463. - * Bazin 463. ${ }^{*}$ C. Lea 463. - ${ }^{*}$ W. Simpson 463. - Pfeffer 463. - E. Lommel 466. Gerland u. Rauwenhoff 467. - Gerland 468. - Baranetzky 471. - Pfeffer 472. - *Referat über die Arbeiten von Gerland, Rauwenhoff u. Lommel 473. - Bert 473. - H. Karsten 474. - A. Batalin 474. - A. Batalin 475. J. Reinke 475. - B. Frank 476. - Heinrich 476. - Raoult 477. - Pleasanton, Poey 477. - Fernere Litteratur 477.

17. Physiologisohe Optik: Lee 481. - M. Schultze 481, - Emmert 481. - Donders 481. - Bähr 482. - Radford 482. - Brachet u. Gsell 482. Strutt 483. - Mott 483. - Lamansky 484. - Maxwell 484. - Dove 485. Airy 486. - Baxt 486. - Rood 487. - Schweigger 487. - Pictet 487. Le Conte 488. - Oppel 489. - Lamansky 489. - Le Conte 489. - Fernere Litteratur 489.

18. Optisch Apparate: Listing 491. - Krippendorf 492. - J. Müller 493. - Stevenson 493. - Williams 493. - Airy 494. - Carrington 494. Buckingham 494. - Loewy 494. - Ertel u. Sohn 495. - Brauer 495. Gruey 496. - Airy 496. - Weiss 497. - Robin 497. - Brachet 497. E. R. L. 497. - Ertel u. Sohn 498. - v. Lang 498. - Southworth 498. Achromatische Linsen 499. - C. A. Young 499. - Schönn 499. - Fleck 500. - Browning, Littrow, Proctor, Young 500. - Sorby 501. - Huggins 502. - Secchi 502. - F. Züllner 503. - D. Kirkwood 503. - Groth 504. Webb 504. - Barthon 504. - Anonym 505. - Morton 505. - Dubosq 505.

- Dudgeon 506. - Fernere Litteratur 506.

\section{Vierter Abschnitt: Wärmelehre.}

19. Theorie der Wärme: Volpicelli 511. - Foncault 511. - Highton 512. - Nicholson 512. - Whiteside Cook 512. - Tyndall 512. - Hudson 512 a. s. w.

Das Druikfehler - Verzeichniss der ersten Hälfte des XXVII. Bandes (1871) der Fortschritte der Physik folgt am Schluss des Bandes.

D. Red.

Verzeichniss der Herren, welche für die erste Hälfte der Fortschritte 1871 Berichte geliefert haben.

Herr Prof. Dr. v. Bezold (Bd.) in München.

- Dr. Erdmann (E. O. E.) in Berlin.

- Prof. Dr. Groth (Gth.) in Strassburg i. E.

- Prof. Dr. Hoppe (He.) in Berlin.

- Prof. Dr. Karsten (K.) in Kiel.

- Dr. Krech (Kr.) in Berlin.

- Dr. Loew (Lw.) in Berlin.

- Prof. Dr. Müttrich (Mch.) in Neustadt E. W.

- Dr. Ohrtmann (O.) in Berlin.

- Prof. Dr. Quincke (Q.) in Heidelberg.

- Prof. Dr. Radicke (Rd.) in Bonn.

- Prof. Dr. Röber (Rb.) in Berlin..

- Prof. Dr. Rüdorff (Rdf.) in Berlin.

- Dr. Saalschütz (Sz.) Königsberg i. Pr.

- Prof. Dr. Schwalbe (Sch.) in Berlin.

- Dr. Wangerin (Wn.) in Berlin.

- Dr. W. Wernicke (W. W.) in Berlin.

- Dr. v. Zahn (Zn.) in Leipzig.

- Prof. Dr. Zöllner (Zr.) in Leipzig. 